\title{
Comparison of Gadoterate Meglumine and Gadobutrol in the MRI Diagnosis of Primary Brain Tumors: A Double-Blind Randomized Controlled Intraindividual Crossover Study (the REMIND Study)
}

\author{
(D).R. Maravilla, (DD. San-Juan, (D).J. Kim, (D) G. Elizondo-Riojas, (D).R. Fink, (DW. Escobar, (D)A. Bag, (DD.R. Roberts, (D). Hao,
} (D) C. Pitrou, (D)A.J. Tsiouris, (DE. Herskovits, and (D).B. Fiebach

\begin{abstract}
BACKGROUND AND PURPOSE: Effective management of patients with brain tumors depends on accurate detection and characterization of lesions. This study aimed to demonstrate the noninferiority of gadoterate meglumine versus gadobutrol for overall visualization and characterization of primary brain tumors.

MATERIALS AND METHODS: This multicenter, double-blind, randomized, controlled intraindividual, crossover, noninferiority study included 279 patients. Both contrast agents (dose $=0.1 \mathrm{mmol} / \mathrm{kg}$ of body weight) were assessed with 2 identical MRIs at a time interval of 2-14 days. The primary end point was overall lesion visualization and characterization, scored independently by 3 off-site readers on a 4-point scale, ranging from "poor" to "excellent." Secondary end points were qualitative assessments (lesion border delineation, internal morphology, degree of contrast enhancement, diagnostic confidence), quantitative measurements (signal intensity), and safety (adverse events). All qualitative assessments were also performed on-site.

RESULTS: For all 3 readers, images of most patients (>90\%) were scored good or excellent for overall lesion visualization and characterization with either contrast agent; and the noninferiority of gadoterate meglumine versus gadobutrol was statistically demonstrated. No significant differences were observed between the 2 contrast agents regarding qualitative end points despite quantitative mean lesion percentage enhancement being higher with gadobutrol $(P<.001)$. Diagnostic confidence was high/excellent for all readers in $>81 \%$ of the patients with both contrast agents. Similar percentages of patients with adverse events related to the contrast agents were observed with gadoterate meglumine (7.8\%) and gadobutrol (7.3\%), mainly injection site pain.
\end{abstract}

CONCLUSIONS: The noninferiority of gadoterate meglumine versus gadobutrol for overall visualization and characterization of primary brain tumors was demonstrated.

ABBREVIATIONS: $\mathrm{AE}=$ adverse event; $\mathrm{CNR}=$ contrast-to-noise ratio; $\mathrm{GBCA}=$ gadolinium-based contrast agent; $\mathrm{LS}$ = least-squares; $\mathrm{rl}=$ relaxivity

adolinium-based contrast agents (GBCAs) have led to im3 provement in the diagnostic accuracy and sensitivity of MR imaging. GBCAs can be classified into linear or macrocyclic agents according to their chemical structure, with macrocyclic GBCAs showing a higher kinetic stability.

Gadoterate meglumine is an ionic macrocyclic GBCA with a

Received May 3, 2017; accepted after revision June 6.

From the Department of Radiology (K.R.M., J.R.F.), University of Washington Medical Center, Seattle, Washington; Clinical Research Department (D.S.-J.), National Institute of Neurology and Neurosurgery, Mexico City, Mexico; Department of Radiology (S.J.K.), Asan Medical Center, University of Ulsan College of Medicine, Seoul, Korea; Universidad Autonoma de Nuevo Leon (G.E.-R.), Hospital Universitario Dr. Jose Eleuterio Gonzalez, Monterrey, Mexico; Centro Medico Imbanaco (W.E.), Cali, Colombia; Department of Radiology (A.B.), University of Alabama, Birmingham, Alabama; Medical University of South Carolina (D.R.R.), Charleston, South Carolina; Guerbet (J.H., C.P.), Roissy-Charles de Gaulle, France; Department of Radiology (A.J.T.), Weill Cornell Medical College, New York, New York; Department of Radiology (E.H.), University of Maryland, Baltimore, Maryland; and Center for Stroke Research Berlin (J.B.F.), Charité-Universitätsmedizin, Berlin, Germany.

This study was supported by Guerbet. measured $\mathrm{T} 1$ relaxivity $(\mathrm{r} 1)$ range in plasma at $37^{\circ} \mathrm{C}$ of $3.4-3.8$ $\mathrm{L} \times \mathrm{mmol}^{-1} \times \mathrm{s}^{-1}$ at $1.5 \mathrm{~T}$ and $3.3-3.7 \mathrm{~L}^{\circ} \mathrm{mmol}^{-1} \times \mathrm{s}^{-1}$ at $3 \mathrm{~T}^{1}$ Gadobutrol is a nonionic macrocyclic GBCA with a higher $\mathrm{r} 1$ range of $4.9-5.5 \mathrm{~L} \times \mathrm{mmol}^{-1} \times \mathrm{s}^{-1}$ at $1.5 \mathrm{~T}$ and $4.7-5.3 \mathrm{~L} \times$ $\mathrm{mmol}^{-1} \times \mathrm{s}^{-1}$ at $3 \mathrm{~T}$ in plasma at $37^{\circ} \mathrm{C} .{ }^{1}$

Gadobutrol is the only GBCA formulated at a concentration of $1 \mathrm{mmol} / \mathrm{mL}$, while gadoterate meglumine is formulated at 0.5 $\mathrm{mmol} / \mathrm{mL}$, as are all other commercially available GBCAs approved for CNS imaging. Both are administered intravenously at a dose of $0.1 \mathrm{mmol} / \mathrm{kg}$ of body weight; thus, gadobutrol is administered at half the volume of gadoterate meglumine. However,

Please address correspondence to Kenneth R. Maravilla, MD, Department of Radiology, University of Washington, 1959 NE Pacific, Box 357115, Seattle, WA 98195; e-mail:kmarav@uw.edu

\footnotetext{
- Indicates open access to non-subscribers at www.ajnr.org

Indicates article with supplemental on-line tables.

Evidence-Based Medicine Level 1.

http://dx.doi.org/10.3174/ajnr.A5316
} 
despite the small differences in relaxivity and in formulated drug concentration, several neuroimaging studies have failed to conclusively demonstrate a clinical advantage for gadobutrol compared with other GBCAs. ${ }^{2-4}$

The primary objective of this study was to determine whether gadoterate meglumine is noninferior to gadobutrol for overall visualization and characterization of primary brain tumors, using an intraindividual crossover comparison design.

\section{MATERIALS AND METHODS \\ Study Design}

This study was a multicenter, double-blind, randomized, controlled intraindividual, 2-sequence, 2-period, crossover noninferiority study. Patients underwent 2 identical MR imaging examinations, 1 with each contrast agent, gadoterate meglumine and gadobutrol. The sequence of contrast agent administration (gadoterate meglumine followed by gadobutrol or vice versa) was randomly assigned to each patient with a 1:1 allocation ratio. This study was conducted according to the principles in the Declaration of Helsinki and the Good Clinical Practices guidelines of the International Conference on Harmonization. In the United States, the protocol was compliant with the Health Insurance Portability and Accountability Act. The study was approved by the local ethics committee or institutional review board for human subject research of each institution in which the study was performed. Written informed consent was obtained from all participating patients. The study was registered on https://clinicaltrials. gov with identifier NCT02034708.

\section{Patients}

A total of 279 adult patients were enrolled in the study at 27 centers worldwide (Mexico, United States, South Korea, and Colombia) between June 2014 and September 2015. Patients with known or highly suspected primary brain (intracranial) tumors detected by previous CT or MR imaging examination and who were scheduled for contrast-enhanced MR imaging were included. Patients were not included if they had a rapidly evolving brain tumor that could change in appearance between the $2 \mathrm{MR}$ imaging examinations or if they were receiving treatment that could result in a change of lesion appearance between the $2 \mathrm{MR}$ imaging examinations (eg, radiation therapy, an operation, or chemotherapy). Additional noninclusion criteria were the following: patients who were pregnant or breastfeeding, had a contraindication to MR imaging, had known severely impaired renal function (estimated glomerular filtration rate of $<30 \mathrm{~mL} / \mathrm{min} / 1.73$ $\mathrm{m}^{2}$ ), had known class III/IV congestive heart failure (New York Heart Association Classification), had a known severe adverse drug reaction or contraindication to GBCA, had received any contrast agent within 48 hours before first study contrast agent injection or were expected to receive any other contrast agent within 24 hours following the last study contrast agent injection, or were included in another clinical trial involving an investigational drug within 30 days before the first contrast agent injection.

\section{Contrast Agent Administration}

Gadoterate meglumine (Dotarem; Guerbet, Aulnay-sous-Bois, France) and gadobutrol (Gadovist, Bayer Schering Pharma, Ber- lin, Germany; or Gadavist, Bayer HealthCare Pharmaceuticals, Wayne, New Jersey) were each administered at a single dose of 0.1 $\mathrm{mmol} / \mathrm{kg}$ of body weight $(0.2 \mathrm{~mL} / \mathrm{kg}$ for gadoterate meglumine, $0.1 \mathrm{~mL} / \mathrm{kg}$ for gadobutrol) by intravenous bolus injection at a rate of $2 \mathrm{~mL} / \mathrm{s}$ with an automatic injector. Contrast agent injection was immediately followed by a saline flush of $20-30 \mathrm{~mL}$ at the same injection rate. To ensure that on-site evaluation of the contrast agent was performed under blinded conditions, a third party performed the injection. The patient was not aware of the identity of the injected contrast agent. The $2 \mathrm{MR}$ imaging examinations were performed with a minimum time interval of 48 hours to allow contrast washout and prevent any effect of contrast agent carryover, and a maximum time interval of 14 days to minimize any potential for lesion change and thus ensure comparability of the 2 examinations.

\section{MR Imaging}

MR imaging was performed on $1.5 \mathrm{~T}$ systems in 15 centers and on 3T systems in 12 centers. Each MR imaging scanner was previously evaluated for qualification for the study by BioClinica (Princeton, New Jersey). It was mandatory that the same MR imaging scanner and same parameters be used for both examinations in each patient. Imaging protocol included 3 unenhanced sequences: axial T1-weighted (2D spin-echo or gradient recalledecho, or TSE with an echo-train length of $\leq 3$ ), axial T2-weighted (2D TSE), and axial T2-weighted FLAIR (2D or 3D FLAIR); and 1 axial T1-weighted sequence post contrast-agent injection acquired with imaging parameters identical to those of the precontrast axial T1 sequence (On-line Table 1). The postcontrast T1weighted imaging was initiated $5 \pm 1$ minutes after the end of the contrast agent injection.

\section{Image Evaluation}

All images were evaluated by 3 independent blinded off-site readers. All were experienced (at least 12 years of experience with an everyday practice in neuroradiology) and analyzed the images for the following qualitative criteria: overall lesion visualization and characterization (as the primary end point), lesion border delineation, visualization of lesion internal morphology, degree of contrast enhancement, and level of diagnostic confidence.

Overall visualization and characterization of the lesion (or most representative lesion, ie, enhancing and/or largest if there was $>1$ lesion present $)$ were assessed on a 4 -point scale $(0=$ poor, inadequate; 1 = fair, partial; 2 = good, adequate; and $3=$ excellent).

The level of diagnostic confidence was evaluated on a 5-point scale according to the percentage of confidence $(1=$ nil, $0 \%-4 \%$; $2=$ poor, $5 \%-35 \%$; $3=$ moderate, $36 \%-65 \% ; 4=$ high, $66 \%-$ $95 \%$; and $5=$ excellent, $96 \%-100 \%$ ) for each patient based on the most representative lesion. All available sequences (FLAIR, T2, and $\mathrm{T} 1$ with and without contrast administration) were available for readers to review and evaluate.

Border delineation and internal morphology were scored on a 3 -point scale ( $0=$ unevaluable; $1=$ seen, but imperfectly; and $2=$ seen completely/perfectly), as was the degree of contrast enhancement $(0=$ nil; $1=$ mild enhancement; and $2=$ strong enhancement). These 3 criteria were assessed on up to 3 of the most 
representative lesions. For each of these 3 criteria, a sum of scores was calculated at the patient level as follows: score lesion 1 $\left(+\right.$ score $_{\text {lesion 2 }}+$ score $_{\text {lesion } 3}$, if applicable) and was recorded as a continuous variable. Sums of scores were therefore related to the number of detected lesions. According to the results for sums of scores, images were classified as "gadoterate meglumine preferred" when the sum of scores of the patient was superior with gadoterate meglumine; as "no preference" when the sums of scores were equal with both contrast agents; and as "gadobutrol preferred" when the sum of scores was superior with gadobutrol.

Off-site readers also performed quantitative measures of signal intensity on up to 3 of the most representative lesions using ROI markers to determine SNR, contrast-to-noise ratio (CNR), and lesion percentage enhancement. ROIs were placed on unenhanced and enhanced $\mathrm{T} 1$ sequences.

The 3 off-site readers were blinded to all patient data and to the time point of the MR imaging examination (first or second), diagnostic results of the imaging procedures, findings of any other readers (ie, investigators, on-site readers, other off-site readers), and the contrast agent administered. Readers were trained on an independent dataset of $15 \mathrm{MR}$ imaging examinations to ensure a harmonized understanding of the reading criteria and were qualified after evaluation of inter- and intrareader variability on 15 new MR imaging examinations.

Study examinations were read in batches of approximately 30 examinations randomly distributed to each reader. Each reader was permitted to analyze a maximum of 1 batch during any given 24-hour period. A "washout" period of at least 2 weeks between examinations from the same patient was applied, and images were distributed in random order between the 2 time points to minimize recall bias.

In conditions more closely simulating the usual practice, an on-site radiologist, blinded to the identity of the contrast agent, also evaluated all images of patients included in his or her site with the same qualitative criteria as described for the blinded readers. These readings were used for patient management.

\section{Safety}

All adverse events (AEs) were recorded from the time of patient enrollment in the study until 30 minutes after the last contrast agent injection. The intensity of each adverse event was graded as mild, moderate, or severe, and the causal relationship to the contrast agent was assessed by the investigator as being not related, doubtfully related, or possibly related. All AEs were classified according to the Medical Dictionary for Regulatory Activities, Version 18.1 (https://www.meddra.org/) index terms.

\section{Statistical Methods}

Statistical analyses were performed by using SAS software (SAS Institute, Cary, North Carolina), Version 9.2.

Primary and secondary criteria were presented on the perprotocol set, including all randomized patients who had a valid off-site assessment of the primary criterion without major protocol deviation. The primary criterion, the noninferiority of gadoterate meglumine-enhanced MR imaging compared with gadobutrol-enhanced MR imaging for overall lesion visualization and characterization (dichotomized as good/excellent versus poor/fair), was assessed with a noninferiority margin set at $-10 \%$, which was considered clinically acceptable. If one assumed that approximately $85 \%$ of patients would have good or excellent lesion visualization and characterization (minimum score of 2 ) for each contrast agent and each independent reader, approximately 235 patients undergoing the $2 \mathrm{MR}$ imaging examinations were needed to ensure that the difference between the 2 examinations did not exceed the noninferiority margin. Assuming that $15 \%$ of patients would withdraw from the study, 270 patients were planned for randomization to achieve the study objective.

The noninferiority of gadoterate meglumine to gadobutrol was declared if the lower limit of the $95 \%$ CI of the difference in overall lesion visualization and characterization was above the noninferiority margin for at least 2 of 3 off-site readers, using a normal test of equality of paired proportions with a 1 -sided $2.5 \%$ significance level. To test the difference between the 2 contrast agents, we performed a multiple logistic regression model for correlated data. Secondary criteria were considered exploratory. Comparison between the 2 contrast agents for continuous variables was performed with the differences of least-squares (LS) mean from a mixed model for correlated data. The frequency of the 3 classifications of sums of scores was compared using the Wilcoxon signed rank test. The intra- and interreader agreement for the evaluation of the overall lesion visualization and characterization was assessed for each contrast agent and presented as agreement proportion. Intrareader agreement was analyzed by rereading $10 \%$ of all MR images, randomly determined. The 3 off-site readers reread the images without knowledge that it was a second reading.

\section{RESULTS}

\section{Patient Characteristics}

A total of 279 patients were enrolled in the study. Five were not randomized due to deviation from the plan specified in the protocol (3 patients) or because they withdrew consent ( 2 patients). A total of 274 patients were randomized to either the gadoterate meglumine/gadobutrol arm (140 patients) or the gadobutrol/ gadoterate meglumine arm (134 patients) to undergo 2 identical MR imaging examinations. Six patients did not receive the first contrast agent, leading to a total of 268 patients receiving at least 1 injection of contrast agent (safety set). Of these, 249 patients underwent a second MR imaging with contrast agent injection and completed the study with both contrast-enhanced MR imaging examinations performed. Patient disposition is summarized in Fig 1.

Among the 249 randomized patients who underwent both MR imaging examinations, 15 were excluded from the per protocol set because they presented with at least 1 major protocol deviation: differences of MR imaging DICOM parameters at T1 sequences between the 2 MR imaging examinations that could adversely affect the comparability of lesion visualization and characterization $(n=3)$, technical injection failure in 1 of the MRIs $(n=3)$, a difference in injection rate of $\geq 1.5 \mathrm{~mL} / \mathrm{s}$ between the 2 contrast agent administrations ( $n=3$ ), a difference of $\geq 10 \%$ between the 2 actual doses administered to a single patient $(n=2)$, patients without detected tumoral lesions according to at least 2 off-site readers at both examinations $(n=3)$, and loss of the second MR 


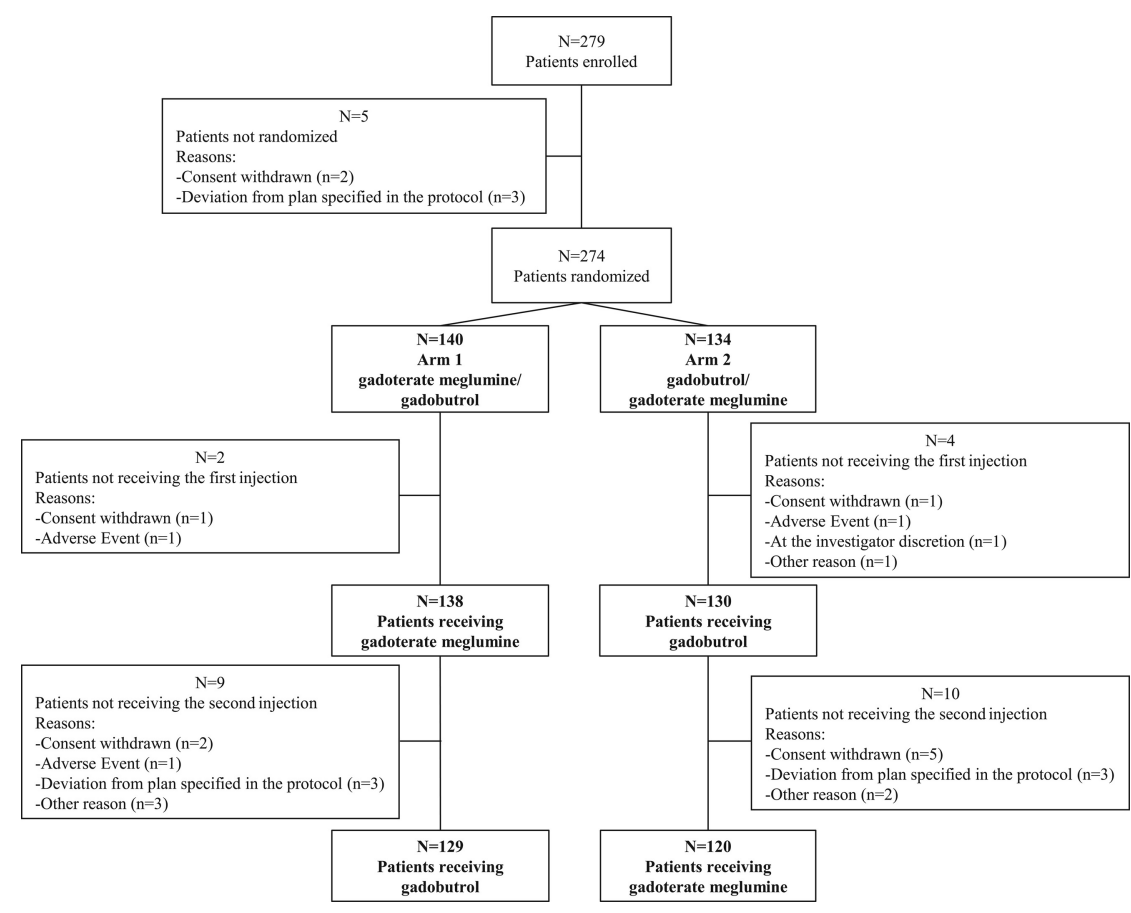

FIG 1. Patient disposition with numbers of patients enrolled $(n=279)$ and randomized $(n=274)$ to undergo 2 identical MR imaging examinations with gadoterate meglumine followed by gadobutrol (arm 1) or with gadobutrol followed by gadoterate meglumine (arm 2). A total of 268 patients received the first contrast agent injection, and 249 patients completed the study with the 2 examinations performed.

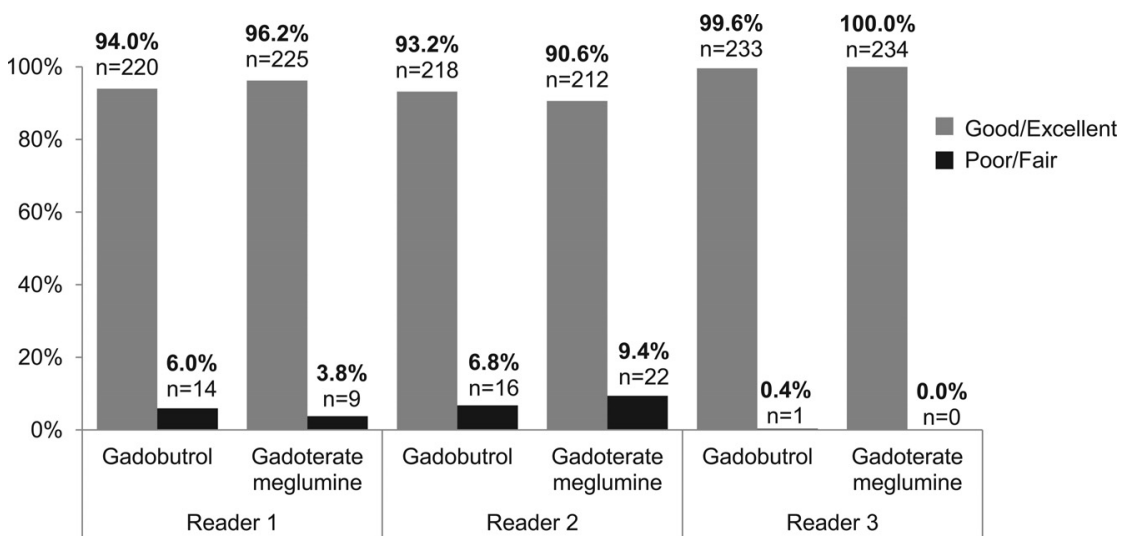

FIG 2. Rate of patients with overall lesion visualization and characterization scored good/excellent or poor/fair. The bar graph shows the rate of patients with overall lesion visualization and characterization scored good/excellent or poor/fair according to each contrast agent and each off-site reader ( $n=234$ patients for all readers).

imaging data $(n=1)$. Therefore, the per-protocol set used for analysis of evaluation criteria included 234 patients, 120 in the gadoterate meglumine/gadobutrol arm and 114 in the gadobutrol/ gadoterate meglumine arm.

The 2 arms showed comparable demographic characteristics (On-line Table 2). Patients ranged in age from 18 to 98 years, with a mean of $53.6 \pm 15.1$ years. There were more female than male patients (64.2\% versus $35.8 \%$ ). Body mass index ranged from 17.4 to $51.2 \mathrm{~kg} / \mathrm{m}^{2}$, with a mean of $27.7 \pm 5.3 \mathrm{~kg} / \mathrm{m}^{2}$. At the time of the last brain imaging evaluation, the patients' diagnoses were tumor of the meninges (49.3\%), glioma (14.9\%), tumor not otherwise specified $(13.1 \%)$, pituitary adenoma $(11.9 \%)$, nerve sheath tumor $(9.0 \%)$, tumor metastases (1.5\%), and other (0.4\%). Among the 268 patients, $170(63.4 \%)$ had a previous examination with GBCA administration and only 1 of these patients $(0.4 \%)$ experienced a nonsevere adverse reaction to the GBCA administered.

\section{Primary Criterion: Overall Lesion Visualization and Characterization}

Figure 2 shows the results of the 3 blinded off-site readers for overall lesion visualization and characterization. For all readers, the overwhelming majority of patients ( $>90 \%$ ) presented with good or excellent overall lesion visualization and characterization with either of the 2 contrast agents. Reader 3 scored overall lesion visualization and characterization as good or excellent for all patients with gadoterate meglumine and for all patients except 1 with gadobutrol. Therefore, a difference between these GBCAs was not statistically evaluable for this reader.

For the 2 other off-site readers, the estimated difference and 95\% CI between gadoterate meglumine and gadobutrol (as reference) was $2.3 \%$ (95\% CI, -1.3 to 5.9 ) for reader 1 and $-2.5 \%$ (95\% CI, -6.5 to 1.4) for reader 2 with lower limits of $95 \%$ CI above the noninferiority limit set at $-10 \%$. The noninferiority of gadoterate meglumine compared with gadobutrol was therefore demonstrated.

Figures 3 and 4 illustrate 2 case examples that demonstrate the identical appearance in lesion visualization and characterization observed for the 2 contrast agents.

Inter- and intrareader agreement for the assessment of overall lesion visualization and characterization was high on the basis of agreement proportion, with interreader agreement ranging from $91 \%$ to $96 \%$ and intrareader agreement, from $96 \%$ to $100 \%$.

The noninferiority of gadoterate meglumine compared with gadobutrol was also demonstrated by on-site readers, with $>96 \%$ of patients presenting good or excellent overall lesion visualization and characterization with both contrast agents and an estimated difference between them of $0.6 \%(95 \%$ CI, -1.8 to 3.1$)$.

\section{Qualitative Secondary Criteria}

The 3 off-site readers scored border delineation, internal morphology, and degree of contrast enhancement on up to 3 of the most representative lesions; a sum of scores was calculated per patient for each of these criteria (On-line Table 3). More than $87 \%$ of patients had only 1 lesion, $5 \%-10 \%$ had 2 lesions, and 


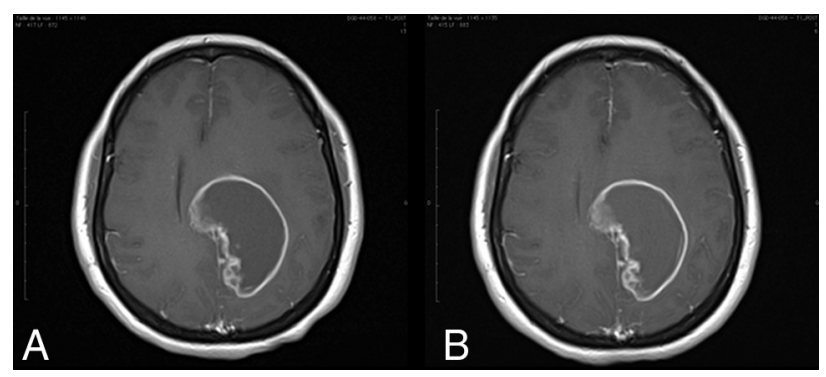

FIG 3. A 46-year-old woman with glioblastoma. $T 1$ spin-echo images (1.5T) after administration of $0.1 \mathrm{mmol} / \mathrm{kg}$ of gadoterate meglumine $(A)$ and gadobutrol $(B)$ show an approximate $65-\mathrm{mm}$ lesion. The time between the 2 MR imaging examinations was 7 days. Lesion visualization and characterization were scored as excellent by all 3 blinded readers. The enhancing rim of the lesion is clearly seen on both examinations.

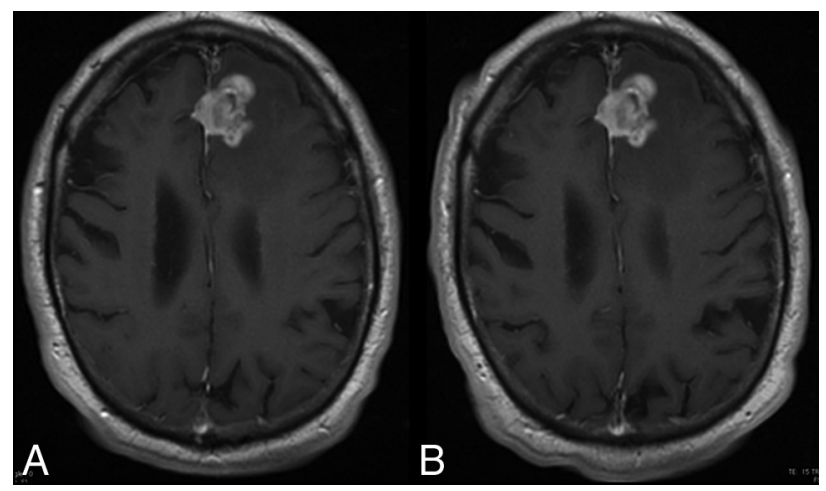

FIG 4. A 74-year-old man with a high-grade glioma. T1 spin-echo images (1.5T) after administration of $0.1 \mathrm{mmol} / \mathrm{kg}$ of gadoterate meglumine $(A)$ and gadobutrol $(B)$ show an approximate $30-\mathrm{mm}$ mass. The time between the 2 MR imaging examinations was 8 days. Lesion visualization and characterization were scored as excellent by all 3 blinded readers. The mass is clearly seen on both examinations and shows no difference in contrast enhancement.

$<4 \%$ had $\geq 3$ lesions. Lesion border delineation showed no significant differences between contrast agents for readers 1 and 2; however, a minimal statistically significant difference $(P=.049)$ was observed in favor of gadobutrol for reader 3. No statistically significant differences between contrast agents were shown for any of the 3 readers for visualization of lesion internal morphology or for qualitative assessment of the degree of lesion contrast enhancement.

The analysis by calculated preference of border delineation, internal morphology, and degree of contrast enhancement between the 2 contrast agents showed no preference of the off-site readers for most patients $(68 \%-89 \%)$ (Fig 5). For the other patients ( $\leq 33 \%$ of patients), a slight calculated preference for gadobutrol was observed for readers 2 and 3, while a slight calculated preference for gadoterate meglumine was seen for reader 1 . These differences were not statistically significant.

The mean level of diagnostic confidence was high or excellent with both GBCAs in $>81 \%$ of patients, according to all off-site readers. No significant difference in diagnostic confidence was observed between the 2 contrast agents.

Similar results were observed between on-site and off-site readers regarding all the qualitative secondary criteria.

\section{Quantitative Secondary Criteria: Signal-Intensity Measurements}

Results of signal-intensity measurements are presented in the Table. LS mean SNR and CNR were higher with gadobutrol than with gadoterate meglumine for all off-site readers, with an LS mean difference ranging from -10.3 to -14.6 for SNR and from -7.4 to -23.6 for CNR. Statistically significant LS mean differences were observed for SNR for reader $1(P<.001)$ and for CNR for readers 1 and $3(P<.001$ and $P=.008)$. Significantly $(P<$ $.001)$ higher values of lesion percentage enhancement were reported with gadobutrol compared with gadoterate meglumine, according to all readers, with LS mean differences ranging from -13.2 to -15.7 .

\section{Adverse Events}

A similar percentage of patients with postinjection AEs related to contrast agent was observed with gadoterate meglumine $(7.8 \%, n=20$ patients $)$ and gadobutrol $(7.3 \%, n=19$ patients). The most frequent AEs related to gadoterate meglumine were injection site pain $(n=11)$ and paraesthesia at the injection site $(n=2)$ and at other sites $(n=2)$, while the most frequent AEs related to gadobutrol were injection site pain $(n=13)$, dizziness $(n=2)$, and nausea $(n=2)$. All these postinjection AEs related to the contrast agents were mild or moderate in intensity and resolved. Two patients had serious AEs during the study ( 1 before and the other 8 days after contrast agent administration). For both patients, the serious AEs were considered related to their primary brain tumor and not related to the contrast agent.

\section{DISCUSSION}

The results from this study support the noninferiority of gadoterate meglumine compared with gadobutrol, 2 macrocyclic GBCAs, for the diagnosis and evaluation of primary brain (intracranial) tumors. Most patients (>90\%) presented with lesions that were scored good/excellent for overall lesion visualization and characterization by the readers with each contrast agent. No statistically significant differences were observed between the 2 GBCAs regarding the assessment of lesion border delineation (except for 1 reader), lesion internal morphology, degree of contrast enhancement, or level of diagnostic confidence. The results for blinded calculated reader preference based on the sum of scores at the patient level for 3 of the secondary end points showed no preference for either contrast agent in most cases $(68 \%-89 \%)$. When a preference was expressed for one or the other contrast agent, the statistical analysis including the no preference subgroup failed to show any statistically significant difference. Despite an LS mean difference in CNR, SNR, and lesion percentage enhancement in favor of gadobutrol, which is considered clinically minimal when relating it to the LS mean value of either of the 2 contrast agents, no discernible clinical effect was observed on lesion visualization and characterization score. This suggests that the level of contrast enhancement obtained with gadoterate meglumine provides comparable contrast visualization for evaluation of primary brain tumors.

Several previous studies compared the efficacy of gadoterate meglumine with that of other GBCAs with slightly different relax- 


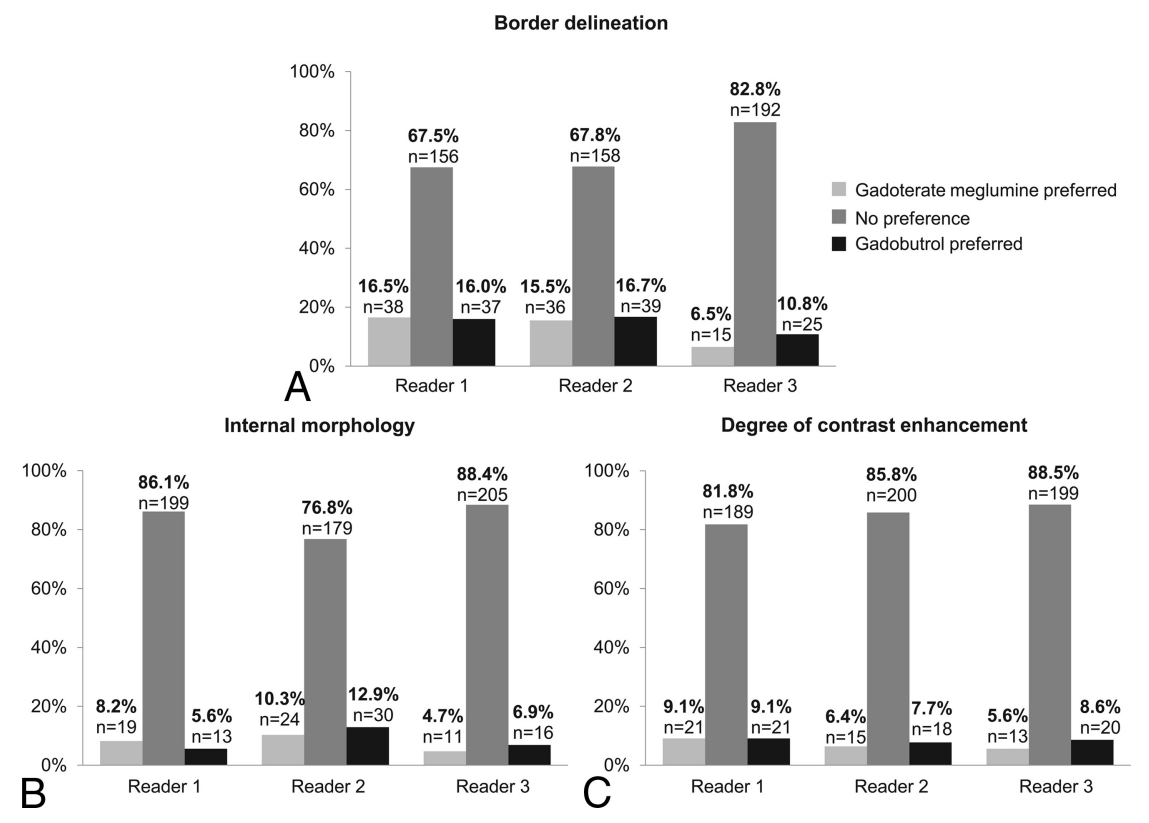

FIG 5. Classification in preference subgroups for lesion border delineation $(A)$, lesion internal morphology (B), and degree of contrast enhancement (C). The bar graphs show the rate of patients classified in preference subgroups for lesion border delineation $(A)$, lesion internal morphology $(B)$, and degree of contrast enhancement $(C)$, according to each off-site reader ( $n=234$ patients for all readers; $n=231$ patients for reader $1 ; n=233$ patients for reader 2; $n=232$ patients for reader 3 ), with $P>.1$ for the 3 end points for all readers (Wilcoxon signed rank test).

\begin{tabular}{|c|c|c|c|c|}
\hline & \multicolumn{2}{|c|}{ LS Mean $^{\mathrm{a}}$ (SE) } & \multirow[b]{2}{*}{$\begin{array}{l}\text { LS Mean }{ }^{\mathrm{a}} \text { Difference } \\
(95 \% \mathrm{Cl})\end{array}$} & \\
\hline & $\begin{array}{l}\text { Gadoterate } \\
\text { Meglumine }\end{array}$ & Gadobutrol & & $P$ Value \\
\hline \multicolumn{5}{|l|}{ SNR } \\
\hline Reader 1 & $266.79(5.00)$ & $281.41(5.02)$ & $-14.61(-22.77$ to -6.46$)$ & $<.001$ \\
\hline Reader 2 & $157.21(4.75)$ & $167.46(4.75)$ & $-10.26(-21.58$ to 1.07$)$ & .076 \\
\hline Reader 3 & $192.94(4.98)$ & $203.35(4.97)$ & $-10.41(-22.75$ to 1.93$)$ & .098 \\
\hline \multicolumn{5}{|l|}{ CNR } \\
\hline Reader 1 & $117.22(7.83)$ & $140.84(7.84)$ & $-23.62(-33.25$ to -13.99$)$ & $<.001$ \\
\hline Reader 2 & $64.59(4.19)$ & $72.02(4.19)$ & $-7.42(-14.87$ to 0.03$)$ & .051 \\
\hline Reader 3 & $82.96(5.16)$ & $96.44(5.15)$ & $-13.48(-23.43$ to -3.54$)$ & .008 \\
\hline \multicolumn{5}{|c|}{ Percentage enhancement } \\
\hline Reader 1 & $107.61(3.93)$ & $121.72(3.93)$ & $-14.12(-18.38$ to -9.85$)$ & $<.001$ \\
\hline Reader 2 & $93.90(3.63)$ & $107.12(3.63)$ & $-13.22(-17.28$ to -9.16$)$ & $<.001$ \\
\hline Reader 3 & $98.82(3.77)$ & $114.47(3.76)$ & $-15.66(-20.52$ to -10.79$)$ & $<.001$ \\
\hline
\end{tabular}

Note:-SE indicates standard error.

${ }^{a}$ LS means are the means controlled for covariates of the model.

ivity values. Oudkerk et $\mathrm{al}^{5}$ compared gadoterate meglumine with gadopentetate dimeglumine, a linear ionic GBCA, in 1038 patients with CNS pathologies and showed comparable image quality with both contrast agents. However, one of the limitations of the study is the lack of a crossover design. More recently, Anzalone et $\mathrm{al}^{2}$ performed a single-blind, crossover comparison study of gadobutrol versus gadoterate meglumine in 136 patients with known brain lesions who underwent 2 identical MR imaging examinations, one with each contrast agent. MR images were read by 3 independent blinded readers in a matched-pairs comparison in which both MR imaging series were displayed simultaneously. Readers scored an overall preference for one or the other MR imaging examination or "no preference" by using a 3-point scale: $1=$ gadobutrol better than gadoterate meglumine, $0=$ no pref- erence, and $-1=$ gadobutrol worse than gadoterate meglumine. Secondary end point evaluations included a preference regarding the intensity of lesion enhancement, lesion delineation, and internal lesion structure. A statistically significant overall preference for gadobutrol was noted by 2 of 3 readers. However, no preference was expressed by the off-site readers in the largest proportion of cases ( $47 \%$ of patients). For secondary assessment, a statistically significant preference in favor of gadobutrol was noted by the 3 readers for the level of enhancement but by only 1 reader for internal lesion structure and by none of the readers for lesion delineation. A major bias of the statistical analysis of this study was that it excluded the "no preference" assessment group, which was the largest group. ${ }^{6}$ Quantitative assessment of the lesion-to-brain ratio and lesion percentage enhancement showed a statistically significant difference in favor of gadobutrol, which probably reflects the small difference in $\mathrm{r} 1$ between the 2 contrast agents. Most important, no clinical advantage for either treatment group was demonstrated in this study, and no differences were reported in the number of lesions detected with either contrast agent.

Gadobutrol was also compared with other GBCAs in several studies. A comparison of the diagnostic performance of gadobutrol and gadoteridol, another macrocyclic agent with $\mathrm{r} 1$ relaxivity comparable with that of gadoterate meglumine, was conducted by Katakami et $\mathrm{al}^{4}$ in 175 patients with known or suspected brain metastases. In this singleblind, randomized, controlled intraindividual crossover study, a single dose and a double dose of gadobutrol, $1.0 \mathrm{~mol} / \mathrm{L}(0.1$ and $0.2 \mathrm{mmol} / \mathrm{kg}$ of body weight, respectively), were demonstrated to be noninferior to a double dose of gadoteridol, $0.5 \mathrm{~mol} / \mathrm{L}(0.2 \mathrm{mmol} / \mathrm{kg}$ of body weight), at detecting brain metastases, with a comparable mean number of lesions detected. A major limitation of this study is that MR imaging examinations with gadoteridol were performed only after the double dose; this feature did not allow a head-to-head comparison of single doses of both contrast agents.

Maravilla et $\mathrm{al}^{3}$ reported a randomized double-blind crossover study also comparing gadobutrol with gadoteridol, both at the single dose of $0.1 \mathrm{mmol} / \mathrm{kg}$. This study, including 229 patients, showed the noninferiority of gadoteridol compared with gadobutrol for overall diagnostic lesion preference using a matchedpairs comparison study design, as well as for secondary end points 
of lesion border delineation, internal morphology, lesion extent, and lesion contrast enhancement. In addition, a blinded reader assessment of the diagnostic characterization of lesions as benign or malignant showed no statistically significant difference between the 2 contrast agents for 2 of 3 readers.

Another limitation of the study is that nearly $60 \%$ of patients presented with extra-axial tumors, meningiomas, or nerve sheath tumors, which should readily enhance regardless of agent and magnetic field strength.

Therefore, the results of the present study are consistent with previous reports showing no difference in clinical benefit between the 2 contrast agents, with slightly different relaxivity values. In our study, a rigorous, double-blind, controlled intraindividual, 2-sequence, 2-period crossover design was chosen to compare gadoterate meglumine with gadobutrol. The study design differs slightly from some other crossover comparison studies because blinded readers used a 4-point scale graded from poor to excellent to rank the primary end point of overall lesion visualization and characterization for each individual MR imaging examination independently and in a randomized order. The present methodology aimed to simulate the clinical daily practice in which contrastenhanced MR imaging must allow good visualization and characterization of lesions, which ensures appropriate medical care of patients. The very high interreader agreement proportion, ranging from $91 \%$ to $96 \%$ for the assessment of overall lesion visualization and characterization, and the consistency observed between off-site and on-site readers demonstrate the robustness of our results.

Unlike in Maravilla et $\mathrm{al}^{3}{ }^{3}$ the accuracy for lesion characterization with histopathologic confirmation was not appraised in our study. The level of diagnostic confidence and radiologic diagnosis was assessed, but histopathologic confirmation ensuring the validity of the diagnosis was lacking. Another limitation of this study was that we did not evaluate the impact of contrast-enhanced MR imaging on patient medical management. A follow-up study evaluating patient management after contrast-enhanced MR imaging with gadoterate meglumine or gadobutrol would be needed to confirm the clinical significance of these results.

The use of any contrast agent must be based on clinical need and diagnostic indications. Once the diagnostic indications are met, the choice of which contrast agent to use is based on balancing the benefit to be gained (eg, adequate visualization) versus the risk of use. Gadoterate meglumine has been used for $>28$ years as a safe and efficient MR imaging GBCA. There have been $>60$ million doses administered worldwide, with an excellent safety profile for immediate adverse drug reactions and a low incidence of serious AEs, comparable with other extracellular GBCAs. ${ }^{7}$ This study confirms the low incidence of immediate AEs reported with gadoterate meglumine and with gadobutrol as demonstrated in multiple previous studies. ${ }^{7,8}$ All AEs were mild or moderate in degree and resolved; no serious AEs related to either contrast agent were reported during the study.

Thus, the present results also support the comparable safety profile of gadoterate meglumine versus gadobutrol.

The discovery of the association of GBCAs with nephrogenic systemic fibrosis in patients with severe renal failure ${ }^{9,10}$ and, more recently, with brain gadolinium deposition ${ }^{1-13}$ in patients with normal renal function raises important safety concerns in the field of contrast-enhanced MR imaging, which influence radiologic practice. Most important, no or very few cases of nephrogenic systemic fibrosis have been reported after highly stable macrocyclic GBCA administration (no cases of nephrogenic systemic fibrosis associated with unconfounded single-agent administration of gadoterate meglumine), and brain T1 hyperintensities have not been observed after administration of any of the macrocyclic GBCAs. ${ }^{12-16}$ The emergence of these safety issues related to the use of GBCAs should encourage the choice of the most stable macrocyclic contrast agents in clinical practice.

\section{CONCLUSIONS}

This multicenter, randomized, double-blind, controlled intraindividual crossover study demonstrates the noninferiority of gadoterate meglumine versus gadobutrol for overall visualization and characterization of primary brain tumors in 234 patients, supported by no preference of the readers for either contrast agent, in most cases, regarding border delineation, internal morphology, and the qualitative degree of contrast enhancement. Despite the difference in relaxivity between these 2 GBCAs, no measurable difference in clinical benefit was observed in our study. A similar safety profile was also demonstrated for both contrast agents.

\section{ACKNOWLEDGMENTS}

The authors thank all participating investigators, Pr K.J. Ahn, Dr A. Arbelaez, Dr E. Caro Osorio, Dr R. Carmody, Dr S.H. Cha, Dr J.W. Choi, Dr G.H. Chung, Dr A.H. Gil Valadez, Dr J.L. Jasso Rico, Dr J.F. Moreno, Dr F. Moser, Dr J. Nickerson, Dr J.A. Romero Figueroa, Dr H. Schwartz, Dr S. Seidman, Dr G. Shah, Dr P. Shah, Dr A.J. Schlesinger, Dr H.R. Vazquez Sanchez, Dr P. Woodard; all study site coordinators; as well as S. Rollin and L. Maho for operational aspects. They also thank B. Piednoir for his statistical input and A.-C. Laurent for her support in editorial assistance.

Disclosures: Kenneth R. Maravilla—RELATED: Grant: Guerbet, Comments: grant for clinical trial study site*; Consulting Fee or Honorarium: Guerbet, Comments: consultant for study design and blinded image reviewer design; Support for Travel to Meetings for the Study or other Purposes: Guerbet, Comments: travel support for consultant meetings; UNRELATED: Consultancy: Bracco, Guerbet. Daniel San-JuanRELATED: Provision of Writing Assistance, Medicines, Equipment, or Administrative Support: Clinical Research Institute, Comments: I received the contrast agents to test in my center.* Sang Joon Kim—RELATED: Grant: Guerbet, Comments: sponsorinitiated trial. James R. Fink—RELATED: Grant: Guerbet, Comments: research salary support (approximately 1\% full time equivalent)*; UNRELATED: Grants/Grants Pending: Guerbet, Comments: research salary support (approximately $1 \%$ full time equivalent)*. William Escobar-RELATED: Consulting Fee or Honorarium: Centro Medico Imbanaco*; UNRELATED: Payment for Lectures Including Service on Speakers Bureaus: Centro Medico Imbanaco. *Asim Bag—RELATED: Grant: Guerbet, Comments: University of Alabama at Birmingham was one of the many sites where the clinical trial was conducted. Guerbet supported all the trial-related expenditures. * Donna R. Roberts—RELATED: Grant: Quintiles/Guerbet, Comments: My university received funding to conduct the clinical trial. I served as site principal investigator*; Support for Travel to Meetings for the Study or Other Purposes: Guerbet, Comments: I received travel support from Guerbet to present the findings of this study at the American Society of Neuroradiology Annual Meeting; UNRELATED: Board Membership: Guerbet, Comments: I serve as a consultant and advisory board member for Guerbet; Consultancy: Guerbet, Comments: I serve as a consultant and advisory board member for Guerbet; Grants/Grants Pending: Guerbet, Comments: grant support for an investigator-initiated study; Payment for Lectures Including Service on Speakers Bureaus: Guerbet, Comments: I have given presentations at scientific meetings. Jing Hao-UNRELATED: Employment: Guerbet, Comments: I am an employee of Guerbet. Camille Pitrou—RELATED: Other: Guerbet, Comments: Guerbet employee; UNRELATED: Employment: Guerbet. Apostolos J. Tsiouris-RELATED: 
Other: BioClinica, Comments: reimbursed for reviewing MRI scans for this study through BioClinica as a blinded independent observer. Edward HerskovitsRELATED: Consulting Fee or Honorarium: BioClinica, Comments: consulting fee for reviewing brain MR imaging examinations; UNRELATED: Board Membership: Galileo CDS, Comments: stock grant for board membership, consulting, software development; Consultancy: Galileo CDS, RadOptimal, Comments: software development, scientific advisor; Expert Testimony: medicolegal consulting; Patents (Planned, Pending or (ssued): Galileo CDS, patent pending; Stock/Stock Options: Galileo CDS, RadOptimal. Jochen B. Fiebach—RELATED: Consulting Fee or Honorarium: Guerbet, Comments: honorarium for blinded reading of study MRI examinations; UNRELATED: Board Membership: European Union Seventh Framework Program (FP7/2007-2013), Comments: grant agreement No. 278276 (WAKE-Up study)*; Consultancy: BioClinica, Cerevast, Artemida, Brainomix, Lundbeck, Comments: consulting and blinded reading of study CT/PET/MRI examinations; Grants/Grants Pending: German Federal Ministry of Education and Research, Comments: Principal Investigator in $01 E O 0801$ and $01 E 101301$. *Money paid to the institution.

\section{REFERENCES}

1. Rohrer M, Bauer H, Mintorovitch J, et al. Comparison of magnetic properties of MRI contrast media solutions at different magnetic field strengths. Invest Radiol 2005;40:715-24 CrossRef Medline

2. Anzalone N, Scarabino T, Venturi C, et al. Cerebral neoplastic enhancing lesions: multicenter, randomized, crossover intraindividual comparison between gadobutrol (1.0M) and gadoterate meglumine $(0.5 \mathrm{M})$ at $0.1 \mathrm{mmol} \mathrm{Gd} / \mathrm{kg}$ body weight in a clinical setting. Eur J Radiol 2013;82:139-45 CrossRef Medline

3. Maravilla KR, Smith MP, Vymazal J, et al. Are there differences between macrocyclic gadolinium contrast agents for brain tumor imaging? Results of a multicenter intraindividual crossover comparison of gadobutrol with gadoteridol (the TRUTH study). AJNR Am J Neuroradiol 2015;36:14-23 CrossRef Medline

4. Katakami N, Inaba Y, Sugata S, et al. Magnetic resonance evaluation of brain metastases from systemic malignances with two doses of gadobutrol $1.0 \mathrm{~m}$ compared with gadoteridol: a multicenter, phase ii/iii study in patients with known or suspected brain metastases. Invest Radiol 2011;46:411-18 CrossRef Medline

5. Oudkerk M, Sijens PE, Van Beek EJ, et al. Safety and efficacy of Dotarem (Gd-DOTA) versus Magnevist (Gd-DTPA) in magnetic resonance imaging of the central nervous system. Invest Radiol 1995;30:75-78 CrossRef Medline

6. Desché P. Re: cerebral neoplastic enhancing lesions-multicenter, randomized, crossover intraindividual comparison between gad- obutrol (1.0M) and gadoterate meglumine $(0.5 \mathrm{M})$ at $0.1 \mathrm{mmol}$ Gd/kg body weight in a clinical setting. Eur J Radiol 2012;81:e967; author reply 2925-26 CrossRef Medline

7. de Kerviler E, Maravilla K, Meder JF, et al. Adverse reactions to gadoterate meglumine: review of over 25 years of clinical use and more than 50 million doses. Invest Radiol 2016;51:544-51 CrossRef Medline

8. Endrikat J, Vogtlaender K, Dohanish S, et al. Safety of gadobutrol: results from 42 clinical phase II to IV studies and postmarketing surveillance after 29 million applications. Invest Radiol 2016;51: 537-43 CrossRef Medline

9. Grobner T. Gadolinium-a specific trigger for the development of nephrogenic fibrosing dermopathy and nephrogenic systemic fibrosis? Nephrol Dial Transplant 2006;21:1104-08 Medline

10. Marckmann P, Skov L, Rossen K, et al. Nephrogenic systemic fibrosis: suspected causative role of gadodiamide used for contrastenhanced magnetic resonance imaging. J Am Soc Nephrol 2006;17: 2359-62 CrossRef Medline

11. Kanda $\mathrm{T}$, Ishii $\mathrm{K}$, Kawaguchi $\mathrm{H}$, et al. High signal intensity in the dentate nucleus and globus pallidus on unenhanced T1-weighted MR images: relationship with increasing cumulative dose of a gadolinium-based contrast material. Radiology 2014;270:834-41 CrossRef Medline

12. Kanda T, Fukusato T, Matsuda M, et al. Gadolinium-based contrast agent accumulates in the brain even in subjects without severe renal dysfunction: evaluation of autopsy brain specimens with inductively coupled plasma mass spectroscopy. Radiology 2015;276: 228-32 CrossRef Medline

13. McDonald RJ, McDonald JS, Kallmes DF, et al. Intracranial gadolinium deposition after contrast-enhanced MR imaging. Radiology 2015;275:772-82 CrossRef Medline

14. Radbruch A, Weberling LD, Kieslich PJ, et al. Gadolinium retention in the dentate nucleus and globus pallidus is dependent on the class of contrast agent. Radiology 2015;275:783-91 CrossRef Medline

15. Radbruch A, Haase R, Kieslich PJ, et al. No signal intensity increase in the dentate nucleus on unenhanced T1-weighted MR images after more than 20 serial injections of macrocyclic gadolinium-based contrast agents. Radiology 2017;282:699-707 CrossRef Medline

16. Cao Y, Huang DQ, Shih G, et al. Signal change in the dentate nucleus on T1-weighted MR images after multiple administrations of gadopentetate dimeglumine versus gadobutrol. AJR Am J Roentgenol 2016;206:414-19 CrossRef Medline 